\begin{abstract}
Reducing $\mathrm{AgNO}_{3}$ by glucose at basic $\mathrm{pH}$ coated the surface of silica spheres with a high density of hemispherical silver nanoparticles (average diameter $3.2 \pm 1 \mathrm{~nm})$. A much lower silver concentration than is standard favored heterogeneous nucleation of silver on the silica surface at the expense of homogeneous nucleation in solution. The slow growth rate of the nuclei promoted the formation of discrete silver particles rather than a continuous shell. Based on scanning electron microscopy and transmission electron microscopy, the surface coverage of silver seed particles was as high as $25 \%$ at $10{ }^{\circ} \mathrm{C}$ without prior functionalization of the silica. The particles were composed of metallic silver based on x-ray photoelectron spectroscopy. There was a sharp increase in the silver surface coverage and decrease in the particle size when the temperature was raised from $5{ }^{\circ} \mathrm{C}$ to $10{ }^{\circ} \mathrm{C}$ and the amount of silica was decreased from 0.2 to $0.025 \mathrm{~V} / \mathrm{V} \%$. The size was controlled by the diffusion barrier through the ion shell surrounding the silica spheres and by maintaining reaction conditions where the particles on the surface compete for silver.
\end{abstract}




\title{
Coating nonfunctionalized silica spheres with a high density of discrete silver nanoparticles
}

\author{
Stephen Purdy \\ Department of Materials Science and Engineering \\ University of Arizona, Tucson, Arizona 85721, USA \\ Anthony J. Muscat* \\ Department of Chemical and Environmental Engineering \\ University of Arizona, Tucson, Arizona 85721, USA
}

Keywords: colloids, nanoparticle, nucleation, Tollens reagent, diammine silver, surface coverage, core-shell

2010 MSC: 00-01, 99-00

\section{Introduction}

Core-shell nanoparticles with a variety of compositions have been studied extensively because of the potential to combine the advantageous properties of two or more materials (Senapati et al., 2014). In particular, much interest 5 has been placed on the coating of dielectric particles with metal nanoparticles (Jiang and Liu, 2003, Jackson and Halas, 2001; Brito-Silva et al., 2013, Peterson et al. 2007). The resulting properties of these composite materials depend largely on their structure (Preston and Signorell, 2009: Tzounis et al., 2014: Pan et al. 2014). For example, the surface plasmon resonance of silver-coated silica particles changes from a single peak to multiple peaks or a single broad peak depending on the connectivity among the silver nanoparticles making up the shell (Peterson et al., 2007, Preston and Signorell, 2009). Similarly, the antimi-

\footnotetext{
${ }^{*}$ Corresponding author

Email address: muscat@email.arizona.edu (Anthony J. Muscat)

URL: http://muscat.chee.arizona.edu/ (Anthony J. Muscat), +1-520-626-6580 (Anthony J. Muscat)
}

Preprint submitted to Journal of Nanoparticle Research

February 14, 2016 
crobial activity of silver particles in the shell depends on their size Markova et al. 2012, Kedziora et al. 2012). Since properties depend on the morphology

15 of the material in the shell, synthesis methods are needed that exert control over the shell material.

Synthesis methods for core-shell silica-silver nanoparticles can be broken down into two broad categories: seeded growth and direct reduction. With seeded growth the silica core is usually functionalized (Jackson and Halas, 2001 ,

20 Kim et al., 2008; Choma et al. 2011), most commonly with either metal ions such as tin (Choma et al. 2012) or an alkoxysilane terminated by either an amine (Kim et al., 2008, Choma et al., 2011, Brinson et al., 2008) or a thiol group (Heuck and Staufer, 2011). The metal nanoparticles are synthesized separately from the silica spheres, and added after the core particle is functionalized. Often the synthesis of the metal nanoparticles includes a ligand or capping agent that makes the interaction between the particle and the silica surface more energetically favorable. Functionalization of the silica particle changes its zeta potential (Goodwin et al., 1990). Under the right conditions, the silica and the metal nanoparticle interact more strongly and adhere to one another. The seed layer doesn't necessarily have to be composed of the same metal as the final coating. In several instances, gold is used as the seed layer, and the final silver shell is grown over the gold seeds by the reduction of a silver salt to metallic silver (Jackson and Halas, 2001, Choma et al., 2012, Dementeva et al., 2012). This method forms bimetallic coatings that have useful properties Kim et al. 35 2008, Zhang et al. 2007).

With direct reduction no seeding step is used. Instead, the metal precursors are reduced at the surface and deposit on the spheres (Zhang et al., 2004), eliminating the need to synthesize the seed particles separately. Similar to the seeded growth method, the surface of the core particles is often functionalized 40 to achieve high surface coverages (Kobayashi et al. 2001; Lee et al., 2006: Zhang et al. 2012). For direct reduction to work, the surfaces of the core particles and precursor ions must be oppositely charged. Otherwise electrostatic repulsion will make the formation of nuclei on the core surface energetically unfavorable. 
Two of the most common chemistries used are the reduction of silver nitrate by polyvinylpyrrolidone (PVP) (Lee et al., 2006) and the reduction of diammine ${ }_{1}$ silver $\left(\left[\mathrm{Ag}\left(\mathrm{NH}_{3}\right)_{2}\right]^{+}\right)$by formaldehyde or glucose (Peterson et al., 2007, Pan et al., 2014, Homan et al., 2010). In the latter method the Tollens' reagent $\left[\mathrm{Ag}\left(\mathrm{NH}_{3}\right)_{2}\right]^{+}$is formed by the complexation of silver ions with ammonia. The complex reacts with aldehydes and alpha hydroxy ketones yielding metallic silver, ammonia, and carboxylic acids.

One drawback of direct reduction is the sparse seed layer that usually forms, which alters the morphology of the final coating. The low nucleation density is the result of either poor attraction between the silver salt and the silica surface or the competition between nucleation and growth. A good understanding of the chemistry and kinetics of seed layer formation is important because it determines whether particles in the final coating remain disconnected or connect and form a porous shell. In this study we show that a dense layer of nanometer-size silver nanoparticles deposit onto much larger silica particles without separately functionalizing or seeding the surface.

\section{Materials and Methods}

Materials

Tetraethoxysilane (TEOS) (Sigma: 86578), A.C.S. grade $\mathrm{NH}_{4} \mathrm{OH}$ (Sigma: 221228), A.C.S. grade $\mathrm{AgNO}_{3}$ (Sigma: 209139), and A.C.S. grade D-(+)-glucose (Sigma: G5767) were purchased from Sigma Aldrich and used without further from ESP chemicals. 18.2 M $\Omega$-cm DI water was used in all processes.

\section{Silica Particle Synthesis}

Silica particles were synthesized using a modified version of the Stöber method (Stöber et al., 1968, Bogush et al., 1988). Briefly, a room temperature solution of water and ammonium hydroxide in ethanol was added to a solution of TEOS in ethanol in a round bottom flask under stirring. The final volume of 
the solution was $111 \mathrm{~mL}$, and was $0.3 \mathrm{M}$ in TEOS, $1 \mathrm{M}$ in $\mathrm{NH}_{4} \mathrm{OH}$, and $8 \mathrm{M}$ in $\mathrm{H}_{2} \mathrm{O}$. The flask was sealed with a ground glass stopper to prevent evaporation of ammonia during the reaction. After $2 \mathrm{~h}$ the resulting particles were centrifuged

75 (4000 RPM, 20 min) and washed 3 times with DI water. The washed particles were dispersed in DI water by sonicating for $10 \mathrm{~min}$. The particle diameter was approximately $500 \mathrm{~nm}$.

\section{Silver Coating}

Silica particles were coated with silver by modifying the procedure described in reference (Peterson et al. 2007$)$. We used a much lower silver concentration at similar ammonia and glucose concentrations and increased both the temperature and reaction time to favor nucleation over particle growth. Coating was performed in a capped plastic Erlenmeyer flask $(250 \mathrm{~mL})$. The reactions were carried out below room temperature by partially submerging the flask in a bath of ice water. First, silica particles suspended in water were added to a glucose solution under moderate stirring and cooled to the desired reaction temperature, which was typically $10{ }^{\circ} \mathrm{C}$. A second solution of $\mathrm{AgNO}_{3}$ and $\mathrm{NH}_{4} \mathrm{OH}$ was added to the mixture of silica particles and glucose to initiate the reaction. Unless otherwise noted, the final solution concentrations were $2 \mathrm{mM} \mathrm{AgNO} 3,33 \mathrm{mM}$ glucose, and $63 \mathrm{mM} \mathrm{NH}_{4} \mathrm{OH}$, with a silica concentration of $0.025 \mathrm{~V} / \mathrm{V} \%$ and a total volume of $50 \mathrm{~mL}$. The reaction was stopped by placing the vessel in an ice bath. The coated particles were separated from the supernatant by centrifugation in a refrigerated centrifuge (Hermle $\mathrm{Z} 400 \mathrm{~K})$ pre-set to $0^{\circ} \mathrm{C}$ at $4000 \mathrm{RPM}$ for 20 min. Washing and centrifugation were repeated 3 times with DI water.

95 Finally, particles were redispersed in ethanol and stored in a refrigerator.

\section{Characterization}

UV-Vis spectra were collected on a Jasco V670 UV-Vis spectrometer. Samples for UV-Vis experiments were dispersed in ethanol and pipetted into a polymethylmethacrylate (PMMA) cuvette. Scanning electron microscopy (SEM) 

the sample degrades to amorphous carbon. The source of the organic material is most likely adsorbed solvent or incompletely hydrolyzed TEOS present in the 

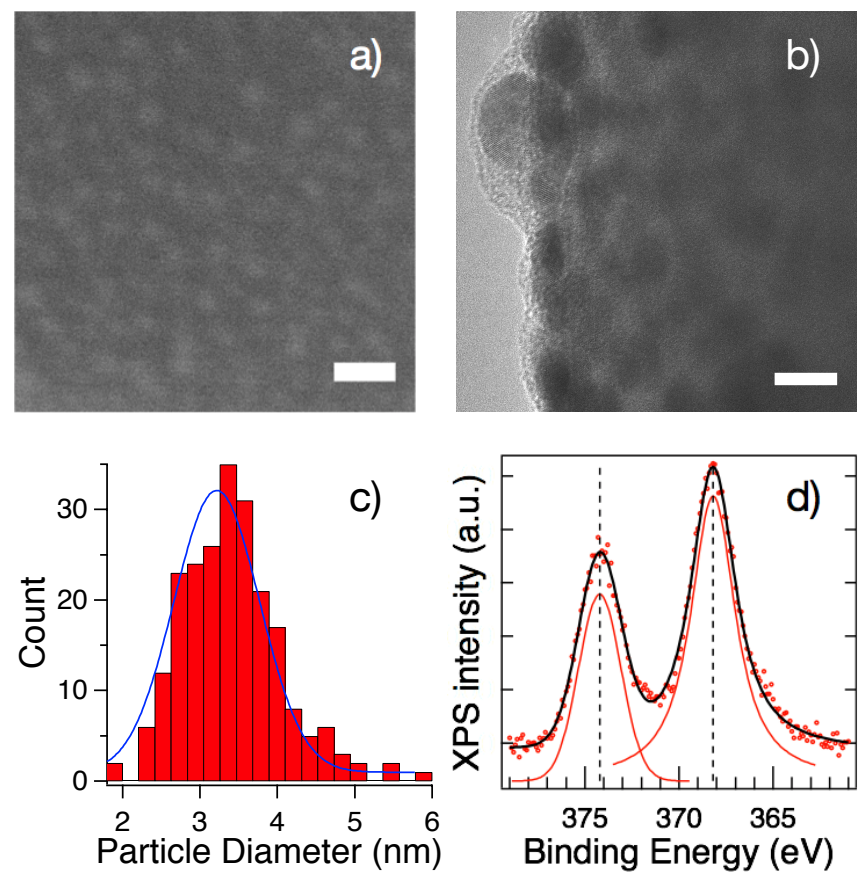

Figure 1: Typical results of the silver coating procedure at $10^{\circ} \mathrm{C}, 2 \mathrm{mM} \mathrm{AgNO} 3,63 \mathrm{mM}$ $\mathrm{NH}_{4} \mathrm{OH}, 33 \mathrm{mM}$ glucose, and $0.025 \mathrm{~V} / \mathrm{V} \%$ silica. Using a low molarity of silver nitrate leads to a high surface coverage of small silver particles $3.2 \pm 1 \mathrm{~nm}$ in size. All scale bars are 25 $\mathrm{nm}$ in length. a) SEM of silica particle surface, b) TEM image of coated silica particle, c) histogram of silver particles from two SEM images, and d) Ag 3d XPS spectrum.

Stöber sphere. Figure 17 shows a histogram of silver particle sizes after a 120 min coating reaction measured from SEM images at $\times 800 \mathrm{k}$ magnification. The sizes of 224 particles were measured from two separate synthesis batches. The average particle size is $3.2 \pm 1 \mathrm{~nm}$. A small number of particles larger than $6 \mathrm{~nm}$ were also present. Figure $1 \mathrm{~d}$ shows the Ag $3 \mathrm{~d}$ XPS spectrum of a silicon wafer containing the silica spheres, confirming the presence of metallic silver on the sphere surface. The two peaks are located at 368.2 and $374.2 \mathrm{eV}$ and assigned to the $\mathrm{Ag} 3 \mathrm{~d}_{5 / 2}$ and $\mathrm{Ag} 3 \mathrm{~d}_{3 / 2}$ states of metallic silver. 


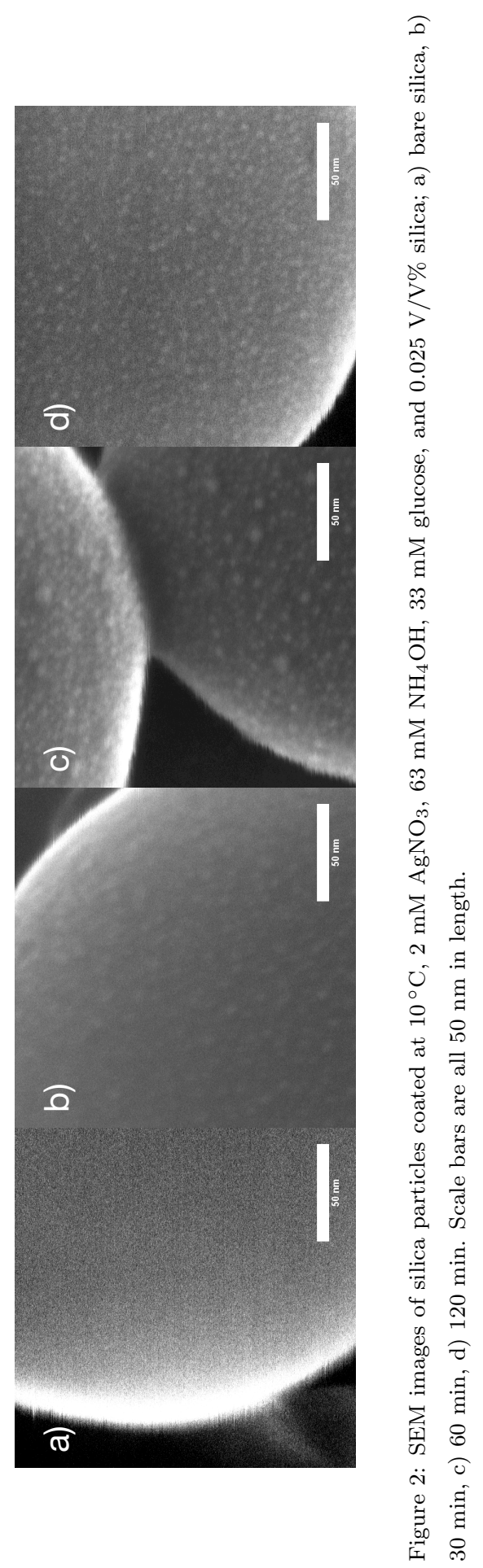




\section{Scanning Electron Microscopy}

Figure 2 shows how the coating morphology changes with reaction time at $10^{\circ} \mathrm{C}$. Samples were imaged before coating (bare silica spheres Figure $2 \mathrm{a}$ ) and

number of silver particles present increases with increasing time. Particles are located almost exclusively on the surface of the silica spheres, and not on the 


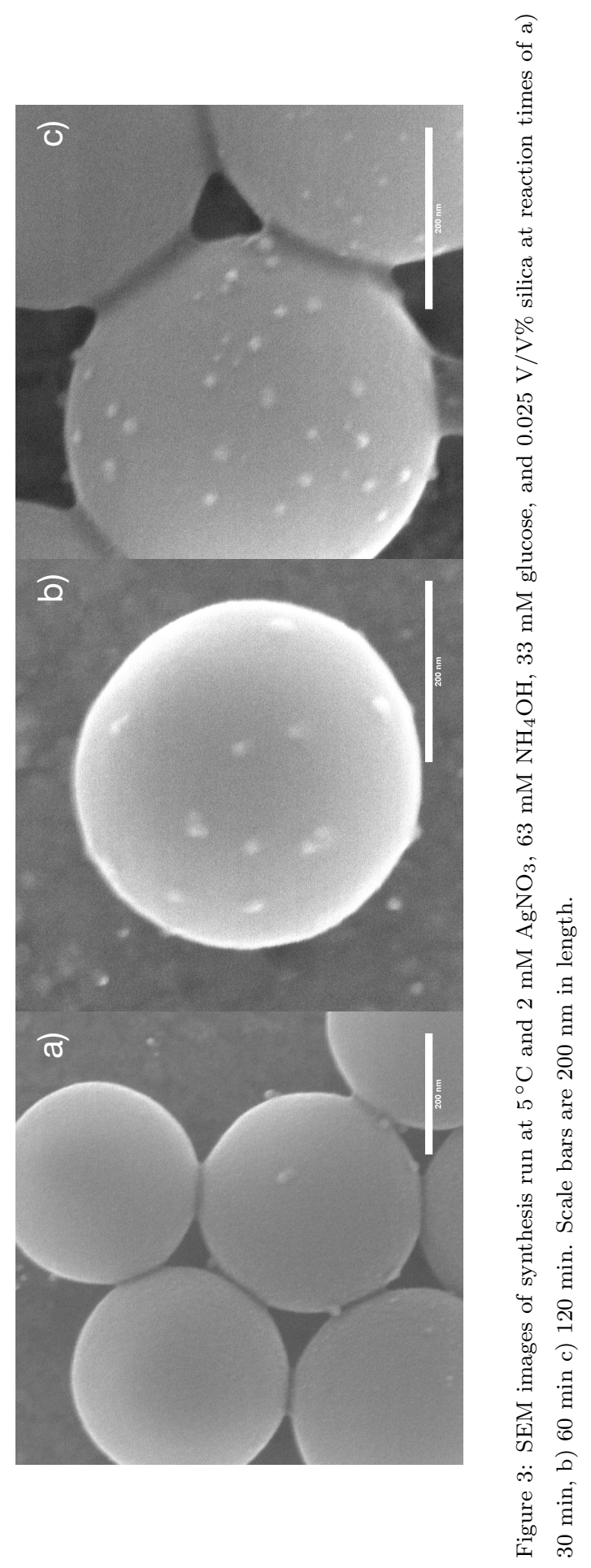


substrate despite three centrifugation and washing cycles prior to preparing the sample for SEM. The few silver particles that were present on the copper SEM substrate are similar in size to the particles present on the silica particle. For the 30 min reaction many spheres had few or no observable silver particles at $\times 100 \mathrm{k}$ magnification. From the few particles observed (14 particles at $\times 150 \mathrm{k}$ magnification) the average diameter was $11.5 \pm 2.9 \mathrm{~nm}$. The change in surface texture typical of the presence of many small silver particles was not present either. Rather the sphere surface was smooth and featureless, bearing more resemblance to the surface texture of the control sample (Figure2a). For the 60 min sample, the average particle size was $11.6 \pm 2.9 \mathrm{~nm}(99$ particles at $\times 150 \mathrm{k}$ magnification). In this sample, the number of particles is higher, but the size and standard deviation are almost identical to the $30 \mathrm{~min}$ sample. For the 120 min sample, the average particle size is $10.1 \pm 2.8 \mathrm{~nm}$ (172 particles measured at $\times 150 \mathrm{k}$ magnification).

Figure 4 shows the surface coverage when the amount of silica particles is varied. All samples were run with the same size silica particles (approximately $500 \mathrm{~nm}$ ) for two hours at $10^{\circ} \mathrm{C}$. The molarities used in the coating process were $2 \mathrm{mM} \mathrm{AgNO} 3,63 \mathrm{mM} \mathrm{NH}_{4} \mathrm{OH}$, and $33 \mathrm{mM}$ glucose. Samples were made at $0.025 \mathrm{~V} / \mathrm{V} \%$ (Figure 4a), $0.05 \mathrm{~V} / \mathrm{V} \%$ (Figure 4b), $0.1 \mathrm{~V} / \mathrm{V} \%$ (Figure 44), and $0.2 \mathrm{~V} / \mathrm{V} \%$ silica (Figure 4 $\mathrm{d}$ ). These values correspond to an increase in surface area from the $0.025 \mathrm{~V} / \mathrm{V} \%$ by a factor of 2,4 , and 8 for the $0.05,0.1$ and $0.2 \mathrm{~V} / \mathrm{V} \%$ samples respectively. At $0.025 \mathrm{~V} / \mathrm{V} \%$, the average particle size was $3.2 \pm 1 \mathrm{~nm}$, with a surface coverage of approximately $25 \%$. Increasing the silica concentration to $0.05 \mathrm{~V} / \mathrm{V} \%$ caused the surface coverage to drop to $9 \%$ with an average particle size of $3.7 \pm 1 \mathrm{~nm}$. The surface coverage at $0.1 \mathrm{~V} / \mathrm{V} \%$ was less than $1 \%$, and at $0.2 \mathrm{~V} / \mathrm{V} \%$ the surface coverage was $0 \%$.

\section{X-ray Photoelectron Spectroscopy}

Figure 5 shows the high resolution XPS spectra of the silver coated silica particles. The $\mathrm{O} 1 \mathrm{~s}$ spectrum has a peak centered at $532.8 \mathrm{eV}$ and a shoulder at $530.2 \mathrm{eV}$. We assign these peaks to O-Si and O-Ag respectively. O-Ag bonds 


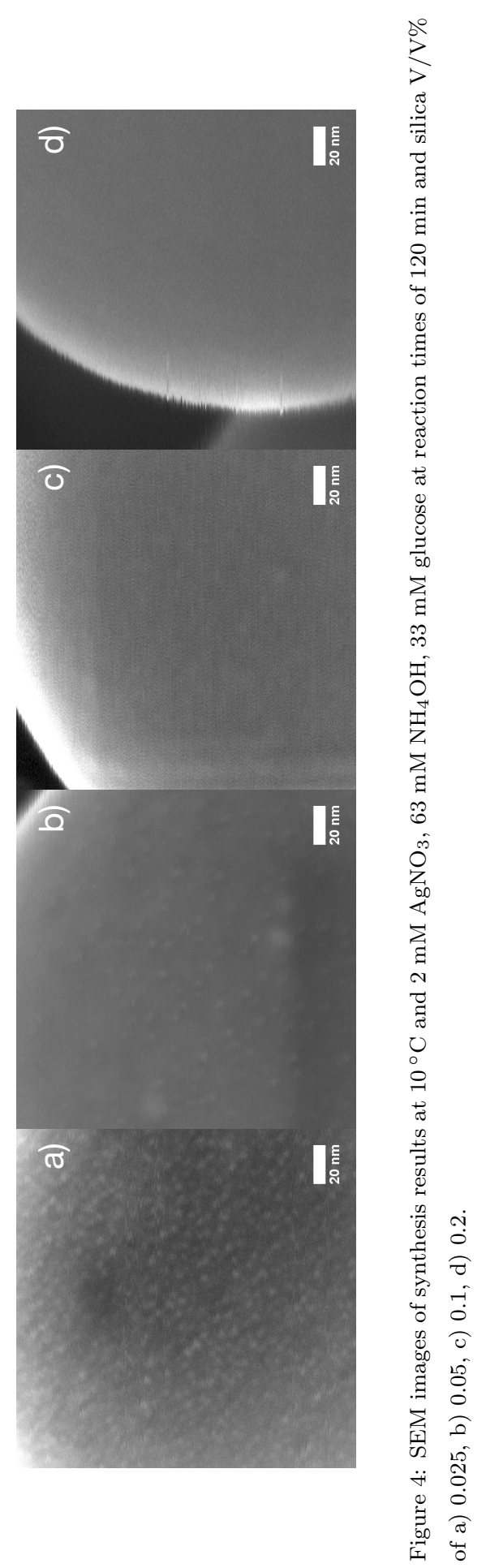


in metallic silver have a binding energy between 528 and $533 \mathrm{eV}$ depending on the configuration (Waterhouse et al., 2004). The Ag 3d spectrum has two peaks centered at $368.2 \mathrm{eV}$ and $374.2 \mathrm{eV}$ that correspond to the $3 \mathrm{~d}_{5 / 2}$ and $3 \mathrm{~d}_{3 / 2}$ states of metallic silver (Moulder et al. 1995). The Si 2p spectrum has two peaks centered at $96.7 \mathrm{eV}$ and $103.2 \mathrm{eV}$. The peak at $103.2 \mathrm{eV}$ corresponds to $\mathrm{SiO}_{2}$ in the Stöber spheres and on any exposed parts of the silicon wafer. The peak at $96.7 \mathrm{eV}$ corresponds to $\mathrm{Si}$, but is shifted to lower binding energy by over $2 \mathrm{eV}$ from where it typically appears at $99.3 \mathrm{eV}$. The large shift is indicative of inhomogeneous charging in this sample. The silicon substrate develops a different surface potential than the silica particles when irradiated by the $\mathrm{x}$ ray source (Kelly, 2003) because the two are not in electrical contact. Thus there are two charging corrections, one for the particles and another for the silicon substrate. We charge corrected for the particles which explains the good agreement between the binding energies of peaks due to the coated particles and values typically found in the literature as well as the low value for the peak resulting from the substrate. Similar phenomenon can be seen in reference (Sohn, 2013).

\section{Discussion}

The presence of silver after the coating reaction is confirmed by the high resolution $\mathrm{Ag} 3 \mathrm{~d}$ and $\mathrm{O}$ 1s XPS spectra. The chemical state of the silver is metallic based on the binding energy of the $\mathrm{Ag} 3 \mathrm{~d}_{5 / 2}$ state. The binding energy of this state for metallic silver is in the narrow range from $368.1-368.3 \mathrm{eV}$, while this peak in silver oxides is shifted to lower binding energies from 367.4-368.0 $\mathrm{eV}$ (Moulder et al. 1995). Because the silver present is metallic, the Ag-O peak is indicative of oxygen in a solid solution with silver or a surface oxygen species. At a binding energy of $530.2 \mathrm{eV}$, our result agrees most closely with oxygen dissolved in the bulk of metallic silver, which has a binding energy between 530.3-530.6 eV (Bao et al., 1996; Waterhouse et al., 2004).

The formation of a dense seed layer by the direct reduction method requires 

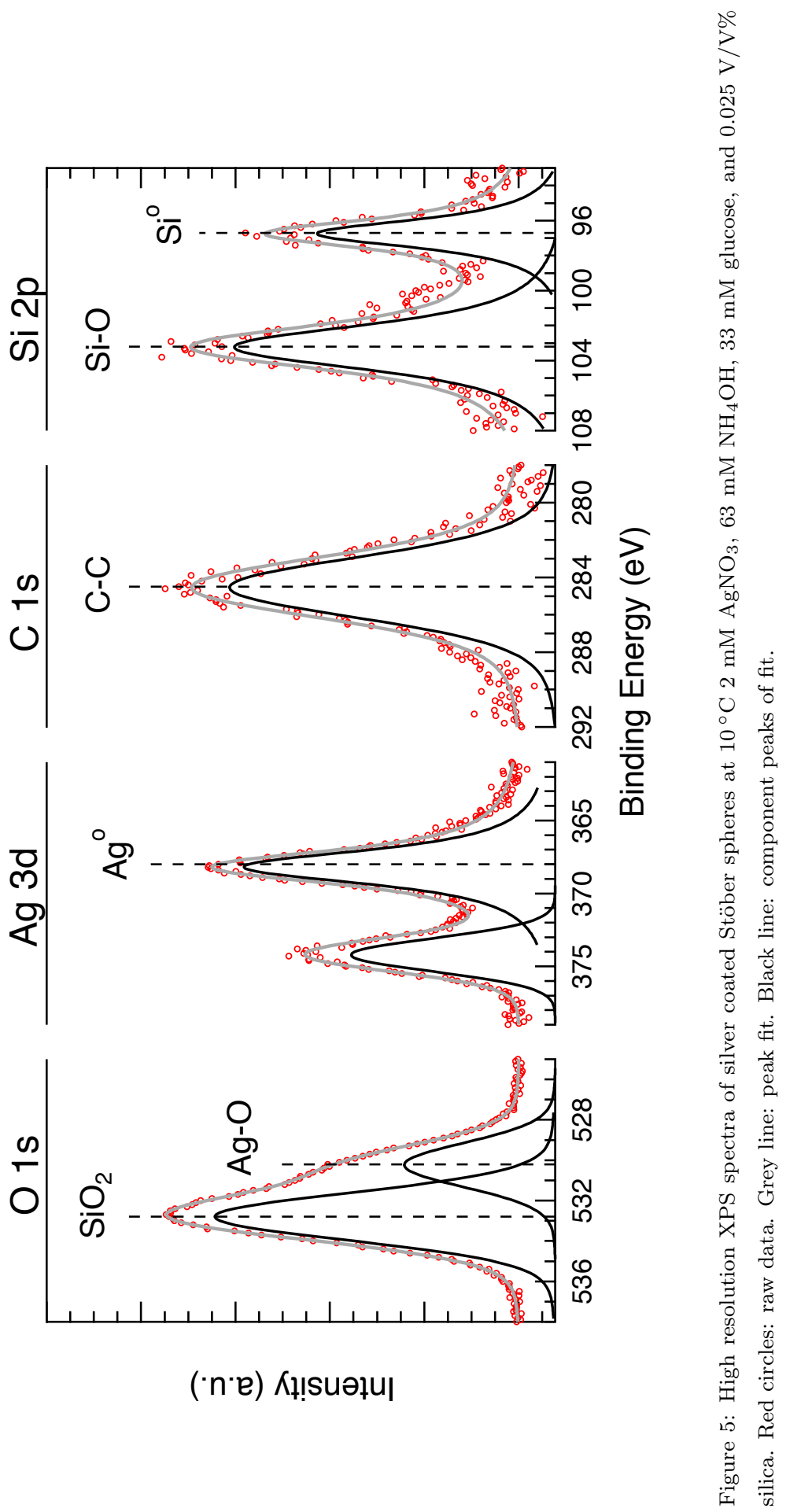
both heterogeneous nucleation and fast nucleation compared to particle growth because the two are in competition for precursors. It is unlikely that under the conditions tested in this study that homogeneous nucleation occurs or that silver nucleates homogeneously and then attaches to the sphere surface. If homogeneous nucleation occurred then we would expect particle agglomeration on the surface, but a relatively monodispersed seed layer was observed by SEM even when nucleation was slowed by lowering the temperature to $5{ }^{\circ} \mathrm{C}$. Homogeneously nucleated silver particles must have precipitated during centrifugation along with the coated silica particles. If this is the case, any homogeneously nucleated silver particles would have been seen in SEM and TEM images as silver particles on the substrate. If any homogeneously nucleated sliver particles remained in the supernatant in even a small concentration, it would have been colored, but in all experiments, the supernatant was clear and colorless. 240 Furthermore, the silver particles were not removed by sonication between centrifugation and washing cycles because few silver particles were found on the copper grid in all of the SEM images taken. The silver was strongly attached to the silica even though the surface was not functionalized and no post synthesis annealing was applied. The TEM micrograph in Figure 1 $\mathrm{b}$ shows the profile of one very large silver particle on the silica surface. The particle is hemispherical in shape with a distinct flat interface between the silver particle and the silica surface, suggesting that the particle nucleated on the silica surface, rather than sticking to the surface after forming in solution.

At an $\mathrm{NH}_{4} \mathrm{OH}$ concentration of $63 \mathrm{mM}$, the $\mathrm{pH}$ of the coating solution is around 11. At this $\mathrm{pH}$, the silica surface is negatively charged (Goodwin et al. 1990). The partial negative charge from the surface hydroxyl groups on the silica surface attracts the diammine silver ions in solution to the surface. When a reducing agent is present, the diammine silver ion is reduced to metallic silver on or close to the silica surface. With a negatively charged surface, it is unlikely that 255 metallic nanoparticles, which are also negatively charged in solution (AlvarezPuebla et al. 2005), would stick to the surface due to electrostatic repulsion. Functionalizing the silica surface to create an amine terminated surface instead 
of a hydroxyl terminated surface changes the zeta potential of the surface enough that nanoparticles in solution can stick to the surface at low to neutral $\mathrm{pH}$ (Park et al. 2006). This change in zeta potential explains why most synthesis methods that use a seeding technique usually functionalize the surface first. This makes the interaction between silica and the seed nanoparticles more energetically favorable which allows for a dense seed layer. As shown in Figure $2 \mathrm{~d}$ many small silver particles nucleated on the silica surface by maintaining a high $\mathrm{pH}$ and slowing the rate of silver reduction. The rate was slowed by reducing the concentrations of $\mathrm{AgNO}_{3}$ and glucose and lowering the temperature of the coating reaction below room temperature.

The effect of temperature is seen in Figures 2 and 3 . The surface coverage is higher and the particles are smaller at $10^{\circ} \mathrm{C}$ than at $5{ }^{\circ} \mathrm{C}$. The change in surface coverage is due to the increased energy barrier to nuclei formation at lower temperature. This energy barrier controls the critical nucleus size, and as the critical nucleus size increases, the nucleation rate decreases (Rempel et al. 2009). Since there are more nuclei present at $10^{\circ} \mathrm{C}$ than at $5{ }^{\circ} \mathrm{C}$ and the availability of precursors and reducing agent is the same, the particles are smaller.

The availability of nucleation sites also plays a role in surface coverage. The number of suitable nucleation sites is directly related to the available surface area. If the silver nanoparticles nucleate evenly across all available surface area, it would follow that an increase in equivalent nucleation sites would cause the particles to be more spread out. This is demonstrated in Figure 4 a $(0.025$ $\mathrm{V} / \mathrm{V} \%$ silica $)$ and Figure $4 \mathrm{~b}(0.050 \mathrm{~V} / \mathrm{V} \%$ silica $)$. The distance between particles grows as the silica concentration is reduced because there are more nucleation sites available. The coverage of silver particles on the surface is reduced as a consequence. Both the average particle size and standard deviation do not 285 change dramatically in the $0.025 \%$ and $0.05 \%$ silica concentrations. From this we conclude that not all nucleation sites have been filled. If growth and nucleation compete for available precursors, one would expect that if all the sites were filled, the surface coverage would reach a maximum and the particles that are 
present would start to grow to a larger size. In the region of silica $\mathrm{V} / \mathrm{V} \%$ tested,

The concentration of $\mathrm{AgNO}_{3}$ and glucose changes the rate of chemical reduction of the diammine silver ion to metallic silver which in turn alters the morphology of the final coating (Brinson et al., 2008). When the solution becomes supersaturated with metallic silver (which occurs at very low concentrations), nuclei of metallic silver form. If this reaction rate is too fast, supersaturation can reach a level that homogeneous nucleation is energetically favorable (Rempel et al. 2009). This is seen when the reaction is carried out at higher $\mathrm{AgNO}_{3}$ concentrations or higher temperatures (Jiang and Liu, 2003). A slow reduction rate allows for the formation of many nuclei on the silica surface that then compete for silver. This competition, along with the diffusion barrier through the ion cloud around the silica particles, leads to the formation of many small silver particles.

\section{Conclusions}

In this paper we demonstrate that a dense layer of small $(3.2 \pm 1 \mathrm{~nm})$ hemispherical silver particles can be grown on the surface of much larger silica spheres without a separate functionalization step. Low concentrations of Tollens' reagent $\left(\left[\mathrm{Ag}\left(\mathrm{NH}_{3}\right)_{2}\right]^{+}\right)$and glucose and low temperature caused silver to nucleate on the silica surface not in the bulk solution and slowed the particle growth rate promoting good surface coverage. The highest surface coverage of discrete nanoparticles was achieved at $10^{\circ} \mathrm{C}$ and a silica concentration of 0.025 $\mathrm{V} / \mathrm{V} \%$. Increasing the amount of silica present during the seeding reaction caused the surface coverage to drop. Lowering the temperature of the reaction from $10{ }^{\circ} \mathrm{C}$ to $5{ }^{\circ} \mathrm{C}$ decreased the surface coverage of silver and increased the particle size. The nanoparticles contained metallic silver as shown by the $\mathrm{Ag}$ $3 \mathrm{~d}_{5 / 2}$ XPS state at $368.2 \mathrm{eV}$. The size and coverage of the silver nanoparticles depend on the temperature, reactant concentrations, and available surface area of silica. 
Peterson et al. used a higher silver concentration at similar ammonia and glucose concentrations and lower temperatures and reaction times than we used because their objective was to promote particle growth and form a continuous metal shell (Peterson et al. 2007). This method was also used by Homan et al. on amine modified silica with varying diammine silver concentrations which changed the silver particle size and surface coverage (Homan et al., 2010). Kobayashi et al. used alternating washes in tin and silver ions to grow a coating 325 of metallic silver by the oxidation of $\mathrm{Sn}^{2+}$ to $\mathrm{Sn}^{4+}$ (Kobayashi et al. 2001). Pan et al. similarly used tin ions adsorbed on silica to grow $10 \mathrm{~nm}$ seeds which were then used to grow a full shell by reduction of Tollens' reagent with glucose (Pan et al., 2014). Jiang et al. found that higher $\mathrm{AgNO}_{3}$ concentrations or temperatures favored homogeneous nucleation (Jiang and Liu, 2003).

The surface coverage of the seed layer could influence the morphology of a shell grown from it. The morphology of the shell material can have an effect on the optical, electronic, and antimicrobial properties of the core shell particle, making control of the seed layer an important part of tuning these properties. Furthermore, small metal nanoparticles have shown catalytic activity that is very different from their bulk counterparts (Lopez et al., 2004). This, along with interactions between a metal catalyst and the substrate on which it is bound, makes core-shell nanoparticles an ideal system to test the combined effects of both particle size and substrate-catalyst interactions at the nanoscale.

\section{Acknowledgments}

The authors thank Pablo Mancheno for his help with collection and analysis of XPS data. The authors also thank Lance Hubbard for his assistance collecting SEM images and for useful conversations about electrostatics in colloidal systems. 


\section{References}

${ }_{345}$ R. A. Alvarez-Puebla, E. Arceo, P. J. G. Goulet, J. J. Garrido, and R. F. Aroca. Role of nanoparticle surface charge in surface-enhanced Raman scattering. $J$. Phys. Chem. B, 109(9):3787-3792, 2005. doi: 10.1021/jp045015o.

X. Bao, M. Muhler, Th. Schedel-Niedrig, and R. Schlögl. Interaction of oxygen with silver at high temperature and atmospheric pressure: A spectroscopic and structural analysis of a strongly bound surface species. Phys. Rev. B, 54: 2249-2262, 1996. doi: 10.1103/PhysRevB.54.2249.

G.H. Bogush, M.A. Tracy, and C.F. Zukoski IV. Preparation of monodisperse silica particles: Control of size and mass fraction. J. Non-Cryst. Solids, 104 (1):95 - 106, 1988. doi: 10.1016/0022-3093(88)90187-1.

B. E. Brinson, J. B. Lassiter, C. S. Levin, R. Bardhan, N. Mirin, and N. J. Halas. Nanoshells made easy: Improving Au layer growth on nanoparticle surfaces. Langmuir, 24(24):14166-14171, 2008. doi: 10.1021/la802049p.

A. M. Brito-Silva, R. G. Sobral-Filho, R. Barbosa-Silva, C. B. de Araújo, A. Galembeck, and A. G. Brolo. Improved synthesis of gold and silver nanoshells. Langmuir, 29(13):4366-4372, 2013. doi: 10.1021/la3050626.

J. Choma, A. Dziura, D. Jamiłoa, P. Nyga, and M. Jaroniec. Preparation and properties of silica-gold core-shell particles. Colloids Surf., A, 373(1-3):167 171, 2011. doi: 10.1016/j.colsurfa.2010.10.046.

J. Choma, D. Jamioła, J. Ludwinowicz, and M. Jaroniec. Deposition of silver nanoparticles on silica spheres and rods. Colloids Surf., A, 411(0):74-79, 2012. doi: http://dx.doi.org/10.1016/j.colsurfa.2012.07.004.

O.V. Dementeva, M.A. Filippenko, K.E. Groman, and V.M. Rudoy. New multifunctional nanoparticles with mesoporous cores and silver shells. Colloid J., 74(4):440-444, 2012. doi: 10.1134/S1061933X12040059. 
J.W. Goodwin, R.S. Harbron, and P.A. Reynolds. Functionalization of colloidal silica and silica surfaces via silylation reactions. Colloid. Polym. Sci., 268(8): 766-777, 1990. doi: 10.1007/BF01411109.

F.C.A. Heuck and U. Staufer. Electroless deposition and structuring of silver electrodes in closed microfluidic capillaries. J. Microelectromech. Syst., 20(2): 451-459, 2011. doi: 10.1109/JMEMS.2011.2105254.

K. Homan, J. Shah, S. Gomez, H. Gensler, A. Karpiouk, L. Brannon-Peppas, and S. Emelianov. Silver nanosystems for photoacoustic imaging and imageguided therapy. J. Biomed. Opt., 15(2):021316-021316-9, 2010. doi: 10.1117/ 1.3365937 .

380 J. B. Jackson and N. J. Halas. Silver nanoshells: Variations in morphologies and optical properties. J. Phys. Chem. B, 105(14):2743-2746, 2001. doi: 10.1021/jp003868k.

Z. Jiang and C. Liu. Seed-mediated growth technique for the preparation of a silver nanoshell on a silica sphere. J. Phys. Chem. B, 107(45):12411-12415, 2003. doi: $10.1021 / \mathrm{jp} 035060 \mathrm{~g}$.

A. Kedziora, W. Strek, L. Kepinski, G. Bugla-Ploskonska, and W. Doroszkiewicz. Synthesis and antibacterial activity of novel titanium dioxide doped with silver. J. Sol-Gel Sci. Technol., 62(1):79-86, 2012. doi: 10.1007/s10971-012-2688-8.

M. A. Kelly. Analyzing insulators with XPS and AES. In D. Briggs and J. T. Grant, editors, Surface Analysis by Auger and X-ray Photoelectron Spectroscopy, pages 191-210. IMPublications, Charlton, 2003.

J. Kim, W. W. Bryan, and T. Randall Lee. Preparation, characterization, and optical properties of gold, silver, and gold-silver alloy nanoshells having silica cores. Langmuir, 24(19):11147-11152, 2008. doi: 10.1021/la8016497. 
Y. Kobayashi, V. Salgueiriño-Maceira, and L. M. Liz-Marzán. Deposition of silver nanoparticles on silica spheres by pretreatment steps in electroless plating. Chem. Mater., 13(5):1630-1633, 2001. doi: 10.1021/cm001240g.

J. Lee, D. Kim, Y. Jun, and S. Oh. Preparation of silica-silver heterogeneous nanocomposite particles by one-pot preparation strategy using polyol process: Size-controlled immobilization of silver nanoparticles. Mater. Res. Bull., 41 (8):1407 - 1416, 2006. doi: j.materresbull.2006.02.010.

N. Lopez, T.V.W Janssens, B.S Clausen, Y. Xu, M. Mavrikakis, T. Bligaard, and J. K. Nørskov. On the origin of the catalytic activity of gold nanoparticles for low-temperature CO oxidation. J. Catal., 223(1):232 - 235, 2004. doi: 10.1016/j.jcat.2004.01.001.

Z. Markova, K. Siskova, J. Filip, K. Safarova, R. Prucek, A. Panacek, M. Kolar, and R. Zboril. Chitosan-based synthesis of magnetically-driven nanocomposites with biogenic magnetite core, controlled silver size, and high antimicrobial activity. Green Chem., 14:2550-2558, 2012. doi: 10.1039/C2GC35545K.

J. F. Moulder, W. F. Stickle, P. E. Sobol, and K. D. Bomben. Handbook of Xray Photoelectron Spectroscopy. Physical Electronics Inc., Eden Prairie, MN, 1995.

K. Pan, Y. Liang, Y. Pu, Y. Hsu, J. Yeh, and H. C. Shih. Studies on the photocatalysis of core-shelled $\mathrm{SiO}_{2}-\mathrm{Ag}$ nanospheres by controlled surface plasmon resonance under visible light. Appl. Surf. Sci., 311(0):399 - 404, 2014. doi: 10.1016/j.apsusc.2014.05.074.

S. Park, M. Park, P. Han, and S. Lee. The effect of pH-adjusted gold colloids on the formation of gold clusters over APTMS-coated silica cores. Bull. Korean Chem. Soc., 27:1341-1345, 2006. doi: 10.5012/bkcs.2006.27.9.1341.

M. S.M. Peterson, J. Bouwman, A. Chen, and M. Deutsch. Inorganic metallodielectric materials fabricated using two single-step methods based on 
the Tollens process. J. Colloid Interface Sci., 306(1):41 - 49, 2007. doi: 10.1016/j.jcis.2006.10.013.

${ }_{425}$ T. C. Preston and R. Signorell. Growth and optical properties of gold nanoshells prior to the formation of a continuous metallic layer. ACS Nano, 3(11):36963706, 2009. doi: 10.1021/nn900883d.

J.Y. Rempel, M.G. Bawendi, and K.F. Jensen. Insights into the kinetics of semiconductor nanocrystal nucleation and growth. J. Am. Chem. Soc., 131

430 (12):4479-4489, 2009. doi: 10.1021/ja809156t.

S. Senapati, S. K. Srivastava, S. B. Singh, and A. R. Kulkarni. SERS active ag encapsulated $\mathrm{Fe} @ \mathrm{SiO}_{2}$ nanorods in electromagnetic wave absorption and crystal violet detection. Environ. Res., 135(0):95-104, 2014. doi: 10.1016/j. envres.2014.08.026.

${ }_{435} \mathrm{Y}$. Sohn. $\mathrm{SiO}_{2}$ nanospheres modified by Ag nanoparticles: Surface charging and CO oxidation activity. J. Mol. Catal. A: Chem., 379(0):59 - 67, 2013. doi: 10.1016/j.molcata.2013.07.015.

W. Stöber, A. Fink, and E. Bohn. Controlled growth of monodisperse silica spheres in the micron size range. J. Colloid Interface Sci., 26(1):62 - 69, 1968. doi: 10.1016/0021-9797(68)90272-5.

L. Tzounis, R. Contreras-Caceres, L. Schellkopf, D. Jehnichen, D. Fischer, C. Cai, P. Uhlmann, and M. Stamm. Controlled growth of Ag nanoparticles decorated onto the surface of $\mathrm{SiO}_{2}$ spheres: A nanohybrid system with combined SERS and catalytic properties. RSC Adv., 4:17846-17855, 2014. doi: $10.1039 / C 4 R A 00121 D$.

G. I. N. Waterhouse, G. A. Bowmaker, and J. B. Metson. Mechanism and active sites for the partial oxidation of methanol to formaldehyde over an electrolytic silver catalyst. Appl. Catal. A-Gen., 265(1):85-101, 2004. doi: 10.1016/j.apcata.2004.01.016. 
J. Zhang, J. Liu, S. Wang, P. Zhan, Z. Wang, and N. Ming. Facile methods to coat polystyrene and flica colloids with metal. Adv. Funct. Mater., 14(11): 1089-1096, 2004. doi: 10.1002/adfm.200400119.

J. Zhang, H. Liu, Z. Wang, and N. Ming. Preparation and optical properties of silica@Ag-Cu alloy core-shell composite colloids. J. Solid State Chem., 180 (4):1291 - 1297, 2007. doi: 10.1016/j.jssc.2007.01.035.

S. Zhang, F. Ren, W. Wu, J. Zhou, L. Sun, X. Xiao, and C. Jiang. Modified in situ and self-catalytic growth method for fabrication of Ag-coated nanocomposites with tailorable optical properties. J. Nanopart. Res., 14(9), 2012. doi: 10.1007/s11051-012-1105-0. 